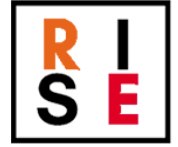

International Journal of Sociology of Education

\section{Hipatia Press}

www.hipatiapress.com

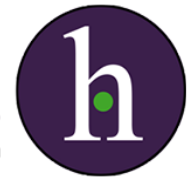

Instructions for authors, subscriptions and further details:

http://rise.hipatiapress.com

\title{
Impactos del confinamiento por el COVID-19 entre universitarios: Satisfacción Vital, Resiliencia y Capital Social Online
}

Antonia Lozano-Díaz ${ }^{1}$

Juan Sebastián Fernández-Prados ${ }^{1}$

Victoria Figueredo Canosa ${ }^{1}$

Ana María Martínez Martínez ${ }^{1}$

1) Universidad de Almería, Spain

To cite this article: Lozano Díaz, A., Fernández-Prados, J.S., Figueredo Canosa, V. \& Martínez Martínez, A.M. (2020 Impactos del confinamiento por el COVID-19 entre universitarios: Satisfacción Vital, Resiliencia y Capital Social Online, International Journal of Sociology of Education, Special Issue: COVID-19 Crisis and Socioeducative Inequalities and Strategies to Overcome them, 79-104. http://doi.org/10.17583/rise.2020.5925

To link this article: http://dx.doi.org/10.17583/rise.2020.5925

PLEASE SCROLL DOWN FOR ARTICLE

The terms and conditions of use are related to the Open Journal System and to Creative Commons Attribution License (CC-BY). 


\section{Impacts of COVID-19}

\section{Confinement among College \\ Students: Life Satisfaction,}

\section{Resilience and Social Capital Online}

Antonia Lozano-Díaz

Universidad de Almería

Victoria Figueredo Canosa

Universidad de Almería
Juan Sebastián Fernández-Prados

Universidad de Almeria, CEMyRI

Ana María Martínez Martínez

Universidad de Almería

(Received: 12 June 2020; Accepted: 19 June 2020; Published: 20 June 2020)

\section{Abstract}

The exceptionality derived from the situation of confinement due to the COVID-19 pandemic has affected all areas of our functioning as a society: relational, health, economic, etc. and of course, education. This study focuses on the impact on these areas and the role played by aspects such as resilience, online social capital and life satisfaction. To this end, an exploratory quantitative study was carried out on 343 university students. The most important conclusion is to note the strong impact that confinement has had on the life situation of students, mainly in the psychological and academic fields, and the important role of the relationship with life satisfaction and resilience. We conclude with the need to work on the issue of resilience from the university and we offer a series of keys to that end.

Keywords: COVID-19, resilience, online social capital, college students 


\section{Impactos del Confinamiento por el COVID-19 entre Universitarios: Satisfacción Vital, Resiliencia y Capital Social Online}

Antonia Lozano-Díaz

Universidad de Almería

Victoria Figueredo Canosa

Universidad de Almería
Juan Sebastián Fernández-Prados Universidad de Almería, CEMyRI Ana María Martínez Martínez Universidad de Almería

(Recibido: 12 Junio 2020; Aceptado:19 Junio 2020; Publicado: 20 Junio 2020)

\section{Resumen}

La excepcionalidad derivada de la situación de confinamiento por la pandemia del COVID-19 ha afectado a todos los ámbitos de nuestro funcionamiento como sociedad: el relacional, el sanitario, el económico, etc. y, por supuesto, el educativo. Este estudio se centra en el impacto en dichos ámbitos y el papel jugado por aspectos como la resiliencia, el capital social online y la satisfacción vital. Para ello se llevó a cabo un estudio cuantitativo de carácter exploratorio sobre 343 estudiantes universitarios. La conclusión más destacada es constatar el fuerte impacto que el confinamiento ha tenido sobre la situación vital del estudiantado en el ámbito psicológico y académico, principalmente, y el importante papel de la relación con la satisfacción vital y la resiliencia, fundamentalmente. Se concluye con la necesidad de trabajar la cuestión de la resiliencia desde la universidad y se dan una serie de claves a tal fin.

Palabras clave: COVID-19, resiliencia, capital social online, alumnado universitario.

2020 Hipatia Press

ISSN: 2014-3575

DOI: $10.17583 /$ rise. 2020.5925 


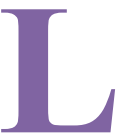

a situación de pandemia generada por el COVID-19 es, sin duda alguna, el evento más extremo al que ha tenido que enfrentarse la sociedad en lo que va de siglo. Tanto por su extensión, que ha afectado a cientos de millones de personas, como por su duración de meses y su previsible continuidad y consecuencias. La situación de excepcionalidad derivada ha abarcado todos los ámbitos de nuestro funcionamiento como sociedad: el relacional, el sanitario, el económico... y, por supuesto, el educativo. El decreto del estado de alarma en España se ha traducido en el confinamiento de millones de personas en sus casas y, siempre que ha sido posible, el traslado de su actividad al formato virtual. En el caso de la universidad ha supuesto, salvo para las universidades virtuales, trasladar el 75\% del estudiantado universitario (Secretaría General de Universidades, 2019) a una versión online de los estudios que hasta el momento cursaban de forma presencial. Una noticia reciente de El País (Silió, 23 de abril de 2020), destaca el estudio de la CRUE que estima en un 3\%, 36.000, el número de estudiantes con problemas técnicos para seguir las clases. El 91\% de los hogares españoles tienen conexión a Internet, el 93\% si hay un joven, por lo que el ministro Castells considera que "los estudiantes tienen más problemas psicológicos o pedagógicos que técnicos".

De este modo, el espacio de lo virtual pasa a convertirse en el nuevo entorno educativo donde el estudiantado debe poner en funcionamiento todas sus estrategias cognitivas, afectivas y motivacionales para autorregular su aprendizaje del modo más exitoso posible (De la Fuente y Lozano-Díaz, 2010). Según Onrubia (2016), un mismo diseño tecno-pedagógico puede suponer modos diferentes de actividad e interactividad en función de momentos y situaciones concretas.

Este trabajo tiene como objetivo general conocer de qué modo ha experimentado el estudiantado universitario la situación de confinamiento. De forma concreta los objetivos serían: 1) conocer qué relaciones existen entre la experiencia vital de confinamiento y la satisfacción vital, la resiliencia y el capital social online; 2) detectar algunas claves de funcionamiento de la satisfacción vital, la resiliencia y el capital social online según perfiles e impactos en la conducta. 


\section{Capital Social}

El concepto de capital social surge a mediados de los años 80 del siglo XX con las teorías de Bourdieu (1986) y Coleman (1988). Desde su origen, diferentes teóricos han centrado sus estudios en este concepto, como Putnam (1993, 1995), Fukuyama (1995), Portes (1998), Lin (1999) y Burt (2004), entre otros.

Desde una perspectiva individual (micro), auspiciada por autores como Bourdieu (1986) y Coleman (1988), el capital social hace referencia a la cantidad y a la calidad de vínculos y redes sociales que un individuo dispone, así como, al conjunto de recursos que puede obtener a través de estos vínculos y redes (López, Civís \& Molina, 2018). Bien sea por herencia o por inversión personal, cada individuo dispone de unos vínculos y redes sociales a partir de los que obtiene recursos instrumentales (ingresos, estatus, etc.) y expresivos (reconocimiento, ayuda mutua, bienestar, etc.), de una forma consciente o inconsciente (Membiela, 2016).

Por otro lado, desde una perspectiva colectiva o comunitaria (macro), suscrita por autores como Putnam (1995), el capital social hace referencia a las relaciones de confianza, las normas y las redes de asociatividad cívica; conducentes a comportamientos cooperativos y prosociales dirigidos a avanzar hacia el bienestar común (Membiela, Sánchez \& Pena, 2019). Se proyecta en la institucionalización de las relaciones de cooperación y reciprocidad, basadas en la confianza, en el marco de las organizaciones, empresas, asociaciones, etc. de una comunidad.

Teniendo en cuenta ambas perspectivas, entendemos por capital social al conjunto de recursos con los que cuenta un individuo o una comunidad, fruto de la confianza entre los miembros y de la formación de redes de asociatividad cívica.

Una vez aclarado el término, es preciso señalar que presenta diferentes dimensiones: estructural, relacional y cognitiva (Nahapiet y Ghoshal, 1998). El capital social estructural se refiere a las conexiones que se producen entre los individuos dentro de la estructura social. El capital social relacional hace referencia a la confianza, la reciprocidad, el apoyo mutuo y el respeto que se genera entre los miembros de la estructura social. Por último, el capital social cognitivo se refiere al entendimiento del conocimiento, normas, valores y 
expectativas compartidas por los miembros de la estructura social (López, Civís \& Molina, 2018).

En la era Internet diferentes medios como son los blogs, foros en webs, redes sociales y similares se han convertido en el espacio de una nueva forma de capital social: el capital social virtual (Nguyen et al., 2013), o capital social online. Así, el uso de las redes sociales virtuales ha contribuido al desarrollo del capital social (Abbas \& Mesch, 2018), jugando un papel clave en el desarrollo de la identidad de los jóvenes (Stokes \& Price, 2017; Heidari et al., 2020), siendo en la actualidad un importante tópico de investigación (Grottke et al., 2018). A nivel de redes, Putnam (2000) establece dos dimensiones de capital social: bonding (vínculo) y bridging (puente). El capital social tipo bonding hace referencia a los recursos a los que se puede acceder a través de los vínculos sociales entre los miembros de grupos homogéneos (que comparten determinadas características relacionadas con la consanguinidad, la etnia, la clase social, la religión, la profesión, etc.). Se trata de vínculos horizontales que brindan un fuerte apoyo social y emocional y acceso a limitados recursos (Abbas \& Mesch, 2018). El capital social tipo bridging se refiere a los recursos a los que se puede acceder a través de los vínculos sociales entre los miembros de grupos heterogéneos que tienen un objetivo o meta común. Se trata de vínculos verticales más débiles que favorecen la conexión con la comunidad en general y proporcionan acceso a recursos más variados (Abbas \& Mesch, 2018).

La influencia de internet en estas dos dimensiones del capital social ha sido abordada en diferentes estudios obteniendo tanto resultados positivos (Abbas \& Mesch, 2018; Grottke et al., 2018) como negativos (Arampatzi et al., 2018; Faucher, 2018); quedando así patente la importancia del uso individual que cada uno hace de internet (Heidari et al., 2020).

\section{Resiliencia}

En el campo de las ciencias sociales la resiliencia es un constructo recurrente en el análisis del éxito académico ante situaciones o contextos difíciles. Se trata de un concepto en torno al cual no existe una definición universalmente aceptada (Brewer et al., 2019; Sandín-Esteban \& Sánchez-Martí, 2015), aunque si existen un cierto consenso en considerarlo como un mecanismo eficaz de superación frente a situaciones adversas (Fergus \& Zimmerman, 2005; McGinnis, 2018; Ramón et al., 2019). El hecho de que la resiliencia se 
considere un aspecto dinámico y afectado por diversas situaciones traslada el debate, según Sandín-Esteban \& Sánchez-Martí (2015), en torno a cuestiones como si la resiliencia es un proceso o un producto, si son factores internos o externos al individuo los que más peso tienen, etc. La mayoría de las investigaciones destacan la importancia de la resiliencia para el equilibrio emocional y el éxito en la universidad por su papel en la superación de situaciones difíciles de tipo emocional, mental o educativo (Cachón et al., 2014; Sandín-Esteban \& Sánchez-Martí, 2015; Sandoval \& Cortés, 2012; Tomás-Miquel et al., 2016).

Por otro lado, existe un consenso amplio entre la comunidad científica sobre la importancia que juega el capital social en la resiliencia ante eventos traumáticos como terremotos (González-Muzzio, 2013), huracanes (Elliot et al., 2009), o cuestiones como el índice de suicidios (Lee 2019). La resiliencia también se relaciona con el optimismo, la satisfacción con la propia vida y el bienestar percibido (Cazalla-Luna \& Molero, 2016; Denovan \& Macaskill, 2017).

Según González-Muzzio (2013), tras un evento traumático aparecen conductas emergentes que movilizan los recursos de los grupos para hacer frente, lo mejor posible, a la nueva situación. Los medios digitales y, sobre todo, las redes sociales facilitan la movilización amplia y diversa del capital social de cada sujeto, y comunidad, para actuar de un modo resiliente mejorando su respuesta, se trata, sin embargo, de un aspecto escasamente investigado en el ámbito concreto de la educación virtual y del alumnado de educación (Brewer et al., 2019).

A raíz de la situación actual de pandemia generada por la COVID-19 han aparecido varios estudios sobre resiliencia como el llevado a cabo sobre el ámbito sanitario (Bryce et al., 2020), el impacto psicológico y social en la vida cotidiana (Arrosi et al. 2020), o el modo en el que la comunidad universitaria desarrolla diferentes estrategias para adaptarse a las nuevas exigencias de la educación virtual (Cleland et al. 2020).

\section{Método}

\section{Diseño}

El estudio que aquí se presenta es un diseño no experimental, de enfoque cuantitativo y de tipo básicamente descriptivo y exploratorio. 


\section{Muestra}

La selección de la muestra fue llevada a cabo de manera incidental entre estudiantes de grado en las facultades de ciencias y ciencias de la educación de la universidad. El cuestionario fue enviado, a través de correo electrónico, a un total de 504 estudiantes de los que completaron todas las preguntas 343, alcanzando una tasa de respuesta del 68\%. Las respuestas fueron recogidas durante un periodo de dos semanas, entre el 11 y el 25 de mayo del presente año. Las dos características sociodemográficas más destacadas de la muestra corresponden a la alta feminización con el $82,8 \%$ del total, debido al peso en la muestra de las participantes de la titulación del grado de educación infantil, y una edad media de $21,8(\mathrm{SD}=5,1)$ distribuida de una manera más o menos homogénea en los tres tramos de edad, 18-19, 20-21 y 22 o más años (ver Tabla 1). Todos los entrevistados de la muestra disponen al menos de un dispositivo conectado a internet (99\% a través del móvil, 95\% en el portátil, $38 \%$ por la Tablet, y solo el $26 \%$ por un PC de sobremesa) y más del $90 \%$ disponen al menos de dos terminales para para mantenerse online, habitualmente móvil y portátil.

Tabla 1

Características sociodemográficas y de conexión de la muestra

\begin{tabular}{ccc}
\hline Sexo & Frecuencia & Porcentaje \\
\hline Hombre & 59 & 17,2 \\
Mujeres & 284 & 82,8 \\
\hline Edad & & 36,2 \\
$18-19$ & 124 & 26,2 \\
$20-21$ & 90 & 37,6 \\
22 o más & 129 & \\
Total & 343 & Porcentaje \\
\hline Dispositivo conectado & Frecuencia & 25,7 \\
\hline Ordenador de sobremesa & 88 & 94,8 \\
Ordenador portátil & 325 & 37,9 \\
Tablet & 130 & 99,1 \\
Móvil & 340 &
\end{tabular}

Fuente: Elaboración propia 
La matriz de datos en formato del paquete estadístico SPSS con toda la información detallada se encuentra disponible para descargar en Mendeley Data con el objeto de favorecer la transparencia y replicabilidad de los análisis (Fernández-Prados y Lozano-Díaz, 2020).

\section{Instrumento}

El instrumento o cuestionario está compuesto por seis bloques:

1. Dos variables sociodemogáficos (sexo, edad)

2. Tres cuestiones relacionadas con la conexión a internet: dispositivo disponible, dispositivo habitual para conectarse y dispositivo habitual para conectarse al aula virtual

3. Tres ítems que recogen una valoración del impacto del confinamiento en su situación vital a través de una escala Likert (1=muy en desacuerdo; $2=$ en desacuerdo; 3=de acuerdo; 4= muy de acuerdo) en base a tres ámbitos de impacto: económico, académico y psicológico:

a) "Mi situación familiar en lo que se refiere a lo socioeconómico ha permanecido igual durante el confinamiento"

b) "Mi rendimiento académico o mi ritmo de trabajo en la universidad ha seguido igual durante el confinamiento"

c) "Mi estado anímico o mi vida emocional, en general, ha continuado igual durante el confinamiento"

4. La Escala de Satisfacción Vital (ESV) compuesta por cuatro ítems está traducida a partir de la Satisfaction with Life Scale (SWLS) diseñada por Cheung y Lucas (2014). Esta escala evalúa el grado de felicidad con la propia vida y está asociada con resultados positivos como una mejor salud, ingresos y rendimiento en el trabajo. Las propiedades psicométricas de la misma concluyen con una fuerte validez del constructo y alta fiabilidad $(\alpha=.90)$

5. La Escala de Resiliencia (ER) empleada en este trabajo es la traducción realizada por Cachón et al. (2020) de la adaptación a su vez de Campbell-Sills, y Stein (2007) que reduce 10 ítems la Connor-Davidson Resilience Scale (CDRISC) compuesta originalmente por 25 ítems (Connor y Davidson, 2003). A pesar de esta reducción drástica de ítems consigue mantener unos coeficientes significativos en el Análisis Factorial Confirmatorio, la validez de constructo y un alfa de Cronbach bastante alto $(\alpha=.85)$ en una escala unidimensional.

6. Por último, la Escala de Capital Social Online (ECSO) contiene también 10 ítems traducidos ad hoc a partir del trabajo de Choi (2018) que contempla 
dos constructos del capital social online: Bridging and Bonding Social Capital, ambos con un coeficiente alfa de Cronbach superior a .92, y que hemos tratado como una sola escala. En cualquier caso, se pueden encontrar la primera subescala (bridging) en los primeros cinco ítems de la ECSO y la segunda (bounding) en los últimos cinco ítems (ver tabla 3).

Tabla 2

Escalas empleadas en el cuestionario

\begin{tabular}{ccc}
\hline $\begin{array}{c}\text { Escalas } \\
\text { (Abreviatura) }\end{array}$ & $\begin{array}{c}\text { Fuente } \\
\text { [adaptación] }\end{array}$ & $\begin{array}{c}\text { Ítems } \\
\text { (coef. Alpha) }\end{array}$ \\
\hline Satisfacción Vital & Cheung y Lucas (2014). & 4 \\
(ESV) & [traducción propia] & $(.90)$ \\
Resiliencia & Campbell-Sills, y Stein (2007) & 10 \\
(ER) & [traducida por Cachón, López, San & $(.85)$ \\
& Pedro, Zagalaz y González (2020)] & 10 \\
Capital social Online & Choi (2018) & $(>.92)$ \\
(ECSO) & [traducción y adaptación propia] & \\
\hline
\end{tabular}

Fuente: Elaboración propia

Todos los ítems de las tres escalas tienen opciones de respuesta en formato Likert y han sido traducidas del inglés al castellano por un experto, salvo la Escala de Resiliencia utilizada y validada en un trabajo reciente sobre una muestra de estudiantes universitarios españoles y chilenos (Cachón et al., 2020). Antes de su administración se llevó a cabo un pilotaje con una decena de estudiantes para intentar detectar errores de comprensión o de cualquier otro tipo. El cuestionario que contiene las diferentes escalas se ha administrado vía correo electrónico mediante la aplicación open source LimeSurvey para encuestas en línea (más información en la página web LimeSurvey: the online surveytool-open source surveys URL: https://www.limesurvey.org).

\section{Análisis de Datos}

El análisis de los datos muestra un conjunto de resultados que van dando respuesta a su vez a los objetivos del estudio, los diferentes análisis y transformación de los datos se detallan como sigue: 
1. En primer lugar, se lleva a cabo un análisis de fiabilidad de las tres escalas ya validadas por sus autores, en este sentido, el trabajo persigue ofrecer instrumentos traducidos y validados para el análisis de datos y también para futuras investigaciones.

2. Se efectúa una transformación lineal de las tres escalas para poder visualizarlas de 0-10. También se describe el comportamiento de las variables sociodemográficas y del impacto del confinamiento en los tres ámbitos considerados que se han dicotomizado por lo que quedarían del modo siguiente:

a) Impacto Económico: "Mi situación familiar en lo que se refiere a lo socioeconómico ha permanecido igual durante el confinamiento" Afectado $=(1=$ muy en desacuerdo; $2=$ en desacuerdo $)$

No afectado $=(3=$ de acuerdo; $4=$ muy de acuerdo $)$

b) Impacto Académico: "Mi rendimiento académico o mi ritmo de trabajo en la universidad ha seguido igual durante el confinamiento" Afectado $=(1=$ muy en desacuerdo; $2=$ en desacuerdo $)$

No afectado $=(3=$ de acuerdo; $4=$ muy de acuerdo $)$

c) Impacto Psicológico: "Mi estado anímico o mi vida emocional, en general, ha continuado igual durante el confinamiento"

Afectado $=(1=$ muy en desacuerdo; $2=$ en desacuerdo $)$

No afectado $=(3=$ de acuerdo; $4=$ muy de acuerdo $)$

3. Se aplicó un análisis de correlaciones a todas aquellas variables continuas del estudio. Básicamente, la matriz de correlaciones permite establecer asociaciones positivas y negativas y la intensidad de las relaciones entre las distintas variables.

4. Finalmente se lleva a cabo un análisis de varianza señala aquellas diferencias de medias significativas de las tres escalas con los distintos valores de las variables sociodemográficas y del impacto o no del confinamiento por ámbitos. 
Tabla 3

Análisis de fiabilidad de las escalas empleadas

\begin{tabular}{|c|c|c|}
\hline Escala de Satisfacción Vital (ESV) & $\begin{array}{c}c \\
(1)\end{array}$ & $\begin{array}{c}\alpha \\
(2)\end{array}$ \\
\hline Las condiciones de mi vida son excelentes & .518 & .821 \\
\hline Mi vida está cerca del ideal & .586 & .779 \\
\hline He conseguido las cosas importantes que quiero en la vida & .515 & .815 \\
\hline Estoy satisfecho con mi vida & .542 & .793 \\
\hline Total & & .844 \\
\hline Escala de Resiliencia (ER) & $\begin{array}{c}\mathrm{c} \\
(1)\end{array}$ & $\begin{array}{c}\alpha \\
(2)\end{array}$ \\
\hline Soy capaz de adaptarme a los cambios & .371 & .848 \\
\hline Puedo afrontar lo que venga & .476 & .840 \\
\hline Veo el lado divertido de las cosas & .407 & .841 \\
\hline Hacer frente al estrés fortalece & .230 & .855 \\
\hline Tiendo a recuperarme tras una enfermedad o dificultad & .361 & .846 \\
\hline $\begin{array}{l}\text { Puedo alcanzar los objetivos que me propongo incluso cuando hay } \\
\text { obstáculos }\end{array}$ & .377 & .845 \\
\hline Aunque esté bajo presión, me centro y pienso con claridad & .373 & .844 \\
\hline No me desanimo fácilmente por los fallos & .448 & .837 \\
\hline Pienso en mí mismo/a como una persona fuerte & .449 & .842 \\
\hline Puedo manejar sentimientos desagradables & .437 & .840 \\
\hline Total & & .857 \\
\hline Escala de Capital Social Online (ECSO) & $\begin{array}{c}\mathrm{c} \\
(1)\end{array}$ & $\begin{array}{c}\alpha \\
(2)\end{array}$ \\
\hline $\begin{array}{l}\text { Interactuar online con personas me hace interesarme en las cosas } \\
\text { que suceden fuera }\end{array}$ & .587 & .803 \\
\hline Interactuar online con la gente me hace querer probar cosas nuevas & .604 & .805 \\
\hline $\begin{array}{c}\text { Conversar online con la gente me hace interesarme en los que } \\
\text { piensan distinto de mí }\end{array}$ & .460 & .806 \\
\hline $\begin{array}{l}\text { Hablar online con la gente me hace sentir curiosidad por otros } \\
\text { lugares del mundo }\end{array}$ & .488 & .809 \\
\hline
\end{tabular}


Tabla 3 (continuación)

\begin{tabular}{ccc}
\hline Escala de Capital Social Online (ECSO) & $\mathrm{c}$ & $\alpha$ \\
& $(1)$ & $(2)$ \\
\hline $\begin{array}{c}\text { Interactuar online con la gente me hace sentir como parte de una } \\
\text { comunidad }\end{array}$ & .348 & .810 \\
$\begin{array}{c}\text { Hay varios compañeros/as en la universidad con los que interactúo } \\
\text { online y confío para resolver mis problemas }\end{array}$ & .488 & .798 \\
$\begin{array}{c}\text { Si tengo problemas técnicos o de conexión en la docencia online, } \\
\text { hay compañeros/as a quienes puedo acudir }\end{array}$ & .570 & .802 \\
$\begin{array}{c}\text { Hay compañeros/as a quien puedo acudir vía online para pedirles } \\
\text { consejo o consultas sobre la marcha las clases virtuales }\end{array}$ & .611 & .798 \\
$\begin{array}{c}\text { Tengo compañeros/as de confianza a los que puedo recurrir online } \\
\text { para encontrar ánimo y apoyo psicológico }\end{array}$ & .595 & .802 \\
$\begin{array}{c}\text { Tengo compañeros/as de confianza a los que puedo recurrir online } \\
\text { para pedirles ayuda económica o material } \\
\text { Total }\end{array}$ & .423 & .821 \\
$\quad \mathbf{8 2 1}$
\end{tabular}

Fuente: Elaboración propia

(1) Correlación múltiple al cuadrado

(2) Alfa de Cronbach si el elemento se suprime

A continuación, se establecieron tres ámbitos para evaluar el impacto del confinamiento a lo largo de los dos últimos meses: el socioeconómico según su situación familiar, académico o ritmo de trabajo y, por último, psicológico entendido como estado anímico y vida emocional. Se interrogó mediante un formato Likert sobre el grado de acuerdo con las afirmaciones que aseveraban permanecer igual en los tres ámbitos (ver Tabla 4). Las tres variables han sido tratadas como continua u ordinal y de manera dicotomizada. Esto es, se ha etiquetado como "afectado" a los que respondieron las opciones 1 y 2 o que estaban "muy en desacuerdo" o "algo en desacuerdo", respectivamente, con que han permanecido igual durante el confinamiento en cada ámbito, y el resto de las alternativas de respuesta se han considerado como "no afectado".

De esta manera, la Tabla 4 presentan los tres ámbitos, por un lado, analizado como variables continuas con sus medias y desviaciones estándares, y por otro, como dicotómica con sus frecuencias y porcentajes. El ámbito psicológico o anímico se considera el más afectado con casi 3 de cada 4 de los encuestados $(73,6 \%)$, seguido del rendimiento académico por más de la mitad $(57,4 \%)$ y el socioeconómico por casi un tercio de los universitarios entrevistados $(31,6 \%)$. No cabe duda de que la crisis del COVID19 ha provocado un gran impacto entre los estudiantes universitarios de la muestra 
cuando el $84,3 \%$ declara estar afectado en al menos uno de los tres ámbitos y un $19 \%$ por los tres, si bien el reconocimiento de que para la amplia mayoría su rendimiento académico es distinto previo al confinamiento debería ser el foco de atención desde el punto de vista académico.

Tabla 4

Descripción del impacto del confinamiento en tres ámbitos

\begin{tabular}{|c|c|c|c|c|}
\hline \multirow[b]{2}{*}{ Ítems (ámbitos) } & \multicolumn{2}{|c|}{$\begin{array}{l}\text { Continua } \\
(1-5)\end{array}$} & \multicolumn{2}{|c|}{$\begin{array}{c}\text { Dicotómica } \\
(\text { afectado }=1+2)\end{array}$} \\
\hline & $\overline{\mathrm{X}}(1)$ & $\sigma(2)$ & $\mathrm{n}(3)$ & $\%$ \\
\hline $\begin{array}{l}\text { Mi situación familiar en lo que se refiere a } \\
\text { lo socioeconómico ha permanecido igual }\end{array}$ & 3,2 & 1,3 & 109 & 31,6 \\
\hline $\begin{array}{l}\text { Mi rendimiento académico o mi ritmo de } \\
\text { trabajo en la universidad ha seguido igual }\end{array}$ & 2,3 & 1,2 & 198 & 57,4 \\
\hline $\begin{array}{l}\text { Mi estado anímico o mi vida emocional, } \\
\text { en general, ha continuado igual }\end{array}$ & 2,0 & 1,1 & 254 & 73,6 \\
\hline $\begin{array}{l}\text { Total } \\
\text { Fuente: Elaboración propia } \\
\text { (1) Media aritmética } \\
\text { (2) Desviación Estándar. } \\
\text { (3) Frecuencia }\end{array}$ & & & 289 & 84,3 \\
\hline
\end{tabular}

Como se ha dicho más arriba, las tres escalas empleadas en el cuestionario se han transformado en un intervalo de 0-10 para una mejor visualización de las descriptivas resultantes según las dos variables sociodemográficas y los tres ámbitos de impacto del confinamiento que a su vez se han dicotomizado. En la Tabla 5 se han presentado las medias aritméticas y desviaciones estándar, donde cabe destacar con las puntuaciones más altas los hombres en las escalas satisfacción vital y resiliencia y, según la edad, lo más jóvenes muestran más satisfacción vital y capital social online, mientras que el grupo de mayor edad tienen una resiliencia más alta.

Sin embargo, más allá de los perfiles sociodemográficos, el resultado más contundente de la descripción de las tres escalas se encuentra cuando las comparamos según la afectación o no en los tres ámbitos. En todos ellos, los afectados han presentado medias aritméticas más bajas en todas las escalas, si bien, las diferencias más amplias se encuentran en la escala de satisfacción vital o ESV, tanto en el impacto económico $(\overline{\mathrm{X}}=4,6$ frente a $\overline{\mathrm{X}}=6,2)$ como en 
el académico ( $\overline{\mathrm{X}}=4,7$ frente a $\bar{X}=5,5)$ y en la escala de resiliencia o ER, concretamente en el impacto psicológico $(\overline{\mathrm{X}}=5,1$ frente a $\overline{\mathrm{X}}=5,9)$.

Tabla 5

Descripción de las escalas según variables sociodemográfica y del impacto del confinamiento

\begin{tabular}{ccccccc}
\hline & \multicolumn{2}{c}{ ESV } & \multicolumn{2}{c}{ ER } & \multicolumn{2}{c}{ ECSO } \\
\hline Sexo & $\overline{\mathrm{X}}(1)$ & $\sigma(2)$ & $\overline{\mathrm{X}}(1)$ & $\sigma(2)$ & $\overline{\mathrm{X}}(1)$ & $\sigma(2)$ \\
\hline Hombre & 5,6 & 2,07 & 5,7 & 1,51 & 6,2 & 1,66 \\
Mujer & 4,9 & 2,10 & 5,2 & 1,47 & 6,2 & 1,75 \\
\hline Edad & $\overline{\mathrm{X}}(1)$ & $\sigma(2)$ & $\overline{\mathrm{X}}(1)$ & $\sigma(2)$ & $\overline{\mathrm{X}}(1)$ & $\sigma(2)$ \\
\hline $18-20$ & 5,1 & 2,11 & 5,1 & 1,49 & 6,4 & 1,54 \\
21 o más & 4,9 & 2,11 & 5,4 & 1,47 & 6,1 & 1,89 \\
\hline I. Económico (3) & $\overline{\mathrm{X}}(1)$ & $\sigma(2)$ & $\overline{\mathrm{X}}(1)$ & $\sigma(2)$ & $\overline{\mathrm{X}}(1)$ & $\sigma(2)$ \\
\hline Afectado & 4,4 & 2,11 & 5,2 & 1,59 & 6,0 & 1,84 \\
No afectado & 5,3 & 2,05 & 5,3 & 1,44 & 6,3 & 1,67 \\
\hline I. Académico (3) & $\overline{\mathrm{X}}(1)$ & $\sigma(2)$ & $\overline{\mathrm{X}}(1)$ & $\sigma(2)$ & $\overline{\mathrm{X}}(1)$ & $\sigma(2)$ \\
\hline Afectado & 4,7 & 2,06 & 5,1 & 1,44 & 6,2 & 1,75 \\
No afectado & 5,5 & 2,08 & 5,6 & 1,51 & 6,3 & 1,70 \\
\hline I. Psicológico (3) & $\overline{\mathrm{X}}(1)$ & $\sigma(2)$ & $\overline{\mathrm{X}}(1)$ & $\sigma(2)$ & $\overline{\mathrm{X}}(1)$ & $\sigma(2)$ \\
\hline Afectado & 4,6 & 2,02 & 5,1 & 1,49 & 6,2 & 1,71 \\
No afectado & 6,2 & 1,92 & 5,9 & 1,32 & 6,4 & 1,80 \\
Total & 5,0 & 2,11 & 5,3 & 1,49 & 6,2 & 1,73 \\
\hline
\end{tabular}

Fuente: Elaboración propia

(1) Media aritmética

(2) Desviación Estándar

(3) Impacto Económico; Impacto Académico e Impacto Psicológico.

Con el objeto de aproximarse a las relaciones entre las variables continuas del estudio, edad, los tres ámbitos de impacto del confinamiento y las tres escalas, se ha creado una matriz de correlaciones (ver Tabla 6). Aunque las correlaciones más altas se han dado, por una parte, entre el impacto académico y psicológico $(\mathrm{r} 343=.42, \mathrm{p}<.01) \mathrm{y}$, por otra, entre la escala de satisfacción vital y resiliencia $(\mathrm{r} 343=.46, \mathrm{p}<.01)$, el interés principal se encuentra entre las escalas y los diferentes ámbitos. Llegados a este punto es conveniente recordar que estos últimos tienen un formato de pregunta con la direccionalidad invertida, es decir cuanto mayor es su cifra, menor es el impacto o está más de acuerdo con que "ha permanecido igual durante el confinamiento". 
Por lo tanto, cabe destacar que la escala de satisfacción vital (ESV) correlaciona positivamente cuanto menor es el impacto en todos los ámbitos con una alta significación $(\mathrm{p}<.001)$, igual ocurre con la escala de resiliencia (ER) aunque con unos coeficientes de correlación algo más bajos, por el contrario, la escala de capital social online (ECSO) solo es significativa ( $p$ $<.01)$ en el ámbito económico.

Tabla 6

Matriz de correlaciones entre las variables continuas del estudio

\begin{tabular}{lccccccc}
\hline & Edad & I. Eco & I. Aca & I. Psi & ESV & ER & ECSO \\
Edad & 1 &,- 042 &,- 064 &, 010 &, 002 &, $122^{*}$ &,- 044 \\
I. Económico &,- 042 & 1 &, $186^{* * *}$ &, $251^{* * *}$ &, $316^{* * *}$ &, $160^{* *}$ &, $156^{* *}$ \\
I. Académico &,- 064 &, $186^{* *}$ & 1 &, $422^{* * *}$ &, $247^{* * *}$ &, $215^{* * *}$ & 0,076 \\
I. Psicológico &, 01 &, $251^{* *}$ &, $422^{* *}$ & 1 &, $379^{* * *}$ &, $265^{* * *}$ & 0,066 \\
ESV &, 002 &, $316^{* *}$ &, $247^{* *}$ &, $379^{* *}$ & 1 &, $464^{* * *}$ &, $255^{* * *}$ \\
ER &, 122 &, $160^{* *}$ &, $215^{* *}$ &, $265^{* *}$ &, $464^{* *}$ & 1 &, $224^{* * *}$ \\
ECSO &,- 044 &, $156^{* *}$ & 0,076 & 0,066 &, $255^{* *}$ &, $224^{* *}$ & 1 \\
\hline
\end{tabular}

Fuente: Elaboración propia

$* \mathrm{p}<.05 * * \mathrm{p}<.01 * * * \mathrm{p}<.001$

Finalmente, se ha llevado a cabo un análisis de varianza para comprobar si aquellas diferencias de medias encontradas en la Tabla 5 pudieran ser significativas o no. Por consiguiente, se ha calculado el estadístico $\mathrm{F}$ de Fisher-Snedecor y su significación para las variables dicotomizadas o factores de sexo, edad y ámbitos de afectación con las tres variables dependientes o escalas del estudio. Aunque las diferencias de medias entre hombres y mujeres resultan significativas en la escala de satisfacción vital y resiliencia, entre los "afectados" y "no afectados" por ámbitos alcanza mayor relevancia. Los tres ámbitos de impacto y afectación o no del confinamiento muestran diferencias muy significativas en sus medias en la escala de satisfacción vital, especialmente el psicológico, $F(1,343)=39.547 ; \mathrm{p}<.001$. Solo entre los afectados y no en los ámbitos académico y psicológico muestran diferencias de media significativas en la escala de resiliencia y de manera destacada de nuevo en el psicológico, $F(1,343)=22.354 ; \mathrm{p}<.001$. Por último, la diferencia de media de la escala de capital social online no es significativa en ninguno de los factores o variables analizadas. 
Tabla 7

ANOVA de factores dicotomizados y escalas como variables dependientes

\begin{tabular}{ccccccc}
\hline & \multicolumn{2}{c}{ ESV } & \multicolumn{2}{c}{ ER } & \multicolumn{2}{c}{ ECSO } \\
\hline Factores & $F(1)$ & $\operatorname{Sig}(2)$ & $F(1)$ & $\operatorname{Sig}(2)$ & $F(1)$ & $\operatorname{Sig}(2)$ \\
Sexo & 6,200 & .013 & 7,439 & .007 &, 000 & .982 \\
Edad (3) & 0,598 & .440 & 2,809 & .095 & 1,694 & .194 \\
I. Económico (3) & 14,079 & .000 & 0,428 & .513 & 2,577 & .109 \\
I. Académico (3) & 15,219 & .000 & 10,267 & .001 & 0,628 & .429 \\
I. Psicológico (3) & 39,547 & .000 & 22,354 & .000 & 0,649 & .421 \\
\hline
\end{tabular}

Fuente: Elaboración propia

(1) F de Fisher-Snedecor

(2) Significación

(3) Variables o factores dicotomizados

\section{Discusión}

Para que se pueda realizar un tipo de estudio donde los alumnos no se queden atrás, especialmente en momentos como el actual, es imprescindible una buena organización en red y un buen trabajo de planificación por parte de todo el claustro docente conjuntamente con su equipo directivo. La experiencia está demostrando que trabajar en línea posee una serie de ventajas que no éramos capaces de visualizar en toda su amplitud. Las posibles deficiencias se han tenido que ir solucionado a medida que se producían y ello ha permitido un perfeccionamiento progresivo de las actividades. En un mundo tan interconectado como el nuestro poner en marcha la competencia de "aprender a aprender" resulta una tarea imprescindible para que el alumno crezca en autonomía y creatividad. El objetivo del centro desde el inicio era claro: "Que cap alumne quede enrere" (Que ningún alumno se quede atrás). El programa escogido por la administración valenciana para que los docentes pudieran trabajar con sus alumnos ha sido el Pla MULAN que incluía la plataforma Moodle de Aules, para el teletrabajo con archivos y corrección, y la Webex meetings con posibles conferencias con los distintos grupos. El Pla MULAN es un método unificado de docencia digital diseñado para impartir, de manera no presencial, las asignaturas y materiales correspondientes a las diversas etapas escolares de la ESO y Bachillerato. Un plan diversificado de las nuevas tecnologías que lo constituyen y con un objetivo único: garantizar la docencia en línea al máximo alumnado posible y ayudar al profesorado en el acceso a estas herramientas. 
Tal y como era de esperar, el estudiantado que se ha mostrado más resiliente es también aquel que menor impacto, por la situación de confinamiento, ha informado a nivel académico y psicológico, los resultados son acordes a los hallados por autores como Cachón et al. (2020), FernándezMartínez et al. (2017), Sandín-Esteban y Sánchez-Martí, (2015), Tomás et al. (2016). La resiliencia muestra elementos comunes con aspectos de la satisfacción vital como el optimismo y la actitud positiva hacia la vida, este trabajo viene a reafirmar dicha relación, en consonancia con estudios como los de Gutiérrez y Romero (2014), Martínez-Martí y Ruch (2017), RamírezFernández et al. (2018), Vizoso (2019). Los resultados arrojados por una y otra escala son casi iguales, a excepción de la ausencia de relación significativa entre la resiliencia y el impacto económico, es decir, los sujetos resilientes lo siguen siendo al margen de la adversidad económica (SandovalHernández y Cortés, 2012). La escasa variabilidad en la edad de la muestra hace que dicha variable no tenga el peso suficiente para mostrarse como significativa en la resiliencia tal y como se apuntaba en el descriptivo inicial de este estudio y se encuentran en otras investigaciones recientes como las de Arrossi et al. (2020). En el caso del sexo, al igual que en otros estudios (Tomyn \& Wemberg, 2018; Vizoso, 2019), los hombres presentan más resiliencia y satisfacción vital que las mujeres. A pesar de ello, esta diferencia entre sexos debe entenderse con reserva dado el alto porcentaje de mujeres en la muestra, el $82,8 \%$ frente al $17,2 \%$ de hombres.

En relación con el capital social online, al igual que en estudios como el de Fernández-Martínez et al. (2017), esta variable correlaciona tanto con la satisfacción vital como con el nivel de resiliencia. Destaca, no obstante, la ausencia de relación con el impacto sobre los ámbitos académico, económico y afectivo. Probablemente sea debido a que no se ha tenido en cuenta el impacto en el ámbito social, en este caso el capital social se ha comportado como una variable que interactúa con la resiliencia (Ramón et al. 2019). Por otro lado, la decisión de considerar el Impacto Académico como "rendimiento académico o ritmo de trabajo", y el Impacto Emocional como "estado anímico o vida emocional", puede haber dado pie a que sean entendidas como opciones terminológicas no unívocas y, con ello, haber podido generar ambigüedad en su comprensión y respuesta afectando así a su relación con el capital social online. En cualquier caso, desde un punto de vista metodológico hay que poner de manifiesto, algunas limitaciones que se han ido apuntando a lo largo del trabajo. Así, la muestra además de incidental tiene un importante sesgo en 
el sexo con una alta feminización. Las escalas empleadas, salvo la de resiliencia, no consta que se hayan empleado en el contexto del estado español y alguno de los ítems posiblemente pueda mejorarse en la traducción. Los análisis aportan una descripción y correlación entre las principales variables, pero no profundiza en cuestiones explicativas ni considera en profundidad algunas variables intervinientes que pudiera ser relevantes como puede ser el sexo o incluso la edad en la resiliencia.

\section{Conclusiones}

La conclusión más destacada de este estudio exploratorio consiste en constatar el fuerte impacto que el confinamiento ha tenido para la situación vital del estudiantado universitario en el ámbito psicológico y académico, principalmente, y la relación existente con la satisfacción vital y la resiliencia, fundamentalmente.

Otro hallazgo, a destacar por su ausencia, es el escaso papel jugado por el capital social online en los ámbitos considerados. Los resultados pueden ser debidos a la ambigua presentación del impacto académico y del impacto emocional. Futuras investigaciones deberán tener en cuenta estos aspectos, así como el poder del capital social online para actuar, o no, como soporte emocional al mismo nivel que lo hace el capital social offline (Williams, 2006). Por otro lado, la vivencia del estudiantado de la muestra como "grupo virtual" quizá no tuvo el margen necesario para desarrollar el suficiente capital social online que actuara como mecanismo de vinculación y con suficiente poder, así, para impactar de modo significativo en los ámbitos comentados. En todo caso, aspectos como el tiempo de rodaje del grupo como "grupo virtual", la edad, el género y una mejor definición de la relación entre las dimensiones del capital social online y la resiliencia deberán ser consideradas en posteriores investigaciones.

Desde un punto de vista global, tanto en las investigaciones sociales aplicadas como en las intervenciones socioeducativas se han incorporado categorías como la satisfacción vital, el capital social y la resiliencia, no solo para analizar o comparar los cambios sociales en distintos países, sino también para saber cómo abordar las situaciones de crisis o incluso catástrofe desde el diseño de políticas más adecuadas. Así, por un lado, los estudios internacionales (Fernández-Prados, Cuenca-Piqueras, González-Moreno, 2019) incluyen estas categorías para planificar y llevar a cabo las políticas 
públicas, concretamente la resiliencia adquiere una dimensión significativa y central en la Estrategia Global de la Unión Europea en un contexto de permanente crisis e inseguridad (Rouet, \& Pascariu, 2019). Por otro, organismos internacionales como la UNESCO y UNICEF, atesoran décadas de experiencia diseñando e implementando planes socioeducativos donde forman a individuos en la resiliencia, programan los currículums y preparan a sus comunidades vulnerables para afrontar riesgos y catástrofes (Selby \& Kagawa, 2014; UNICEF, 2019). La revisión más detenida de estos estudios internacionales especialmente en Europa y las experiencias de los organismos internacionales en el ámbito educativo en relación particularmente a la categoría de resiliencia, será un reto de próximos trabajos de investigación para precisar conexiones teóricas, sus dimensiones e ítems y orientar posibles desarrollos educativos.

Dados estos resultados y conclusiones, no es descabellado proponer, al igual que hacen otros autores como Brewer (2019), Vizoso (2019) o Woods (2016), la importancia de incorporar la formación en resiliencia del alumnado universitario. En este sentido, las últimas aportaciones consideran que la clave de un cambio hacia la resiliencia es la reflexión sobre uno mismo y la comprensión de quién se es y lo que se quiere ser, por lo que la formación debería centrarse en cuestiones como: el fortalecimiento del autoconcepto y la percepción de autoeficacia (Beltman et al. 2018; Castro et al. 2018; Hazel, 2018; Noble \& McGrath, 2018) y/o en la identificación de los puntos débiles y fuertes para mejorar la capacidad de resiliencia (Beltman et al. 2018; Wosnitza et al. 2018).

\section{Referencias}

Abbas, R., \& Mesch, G. (2018). Do rich teens get richer? Facebook use and the link between offline and online social capital among Palestinian youth in Israel. Information, Communication \& Society, 21(1), 63-79. https://doi.org/10.1080/1369118X.2016.1261168

Arampatzi, E., Burger, M. J., \& Novik, N. (2018). Social network sites, individual social capital and happiness. Journal of Happiness Studies, 19(1), 99-122. https://doi.org/10.1007/s10902-016-9808-z

Arrossi, S., Ramos, S., Paolino, M., Binder, F., Perelman, L. \& Kruptizki, H. (2020). Estudio TIARA. Primer avance de resultados. Centro de 
Estudios de Estado y Sociedad (CEDES)

http://repositorio.cedes.org/handle/123456789/4534

Beltman, S., Mansfield, C., Wosnitza, M., Weatherby-Fell, N. \& Broadley

T. (2018). Using Online Modules to Build Capacity for Teacher

Resilience. En Wosnitza, M., Peixoto, F., Beltman, S. \& Mansfield, C.

F. (Eds.), Resilience in Education. Concepts, Contexts and

Connections (pp. 237-254). Springer.

Bourdieu, P. (1986). Handbook of theory and research for the sociology of education. In J. Richardson (Ed.), Forms of capital, (241-258).

Greenwood Press.

Brewer, M.; van Kessel, G.; Sanderson, B.; Naumann, F.; Lane, M.;

Reubenson, A. \& Carter, A. (2019). Resilience in higher education students: a scoping review. Higher Education Research \&

Development, 38(6), 1105-1120.

https://doi.org/10.1080/07294360.2019.1626810

Bryce, C., Ring, P., Ahsby, S., \& Wardman, J. K. (2020). Resilience in the face of uncertainty: early lessons from the COVID-19 pandemic. Journal of Risk Research.

https://doi.org/10.1080/13669877.2020.1756379

Burt, R. S. (2004). Structural Holes and Good Ideas. American Journal of Sociology, 110(2), 349-399. https://doi.org/10.1086/421787

Cachón, J., López, I., San Pedro, M. B., Zagalaz, M. L., \& González, C. (2020). The Importance of the Phoenix Bird Technique (Resilience) in Teacher Training: CD-RISC Scale Validation. Sustainability, 12(3), 1002. https://doi.org/10.3390/su12031002

Campbell-Sills, L., \& Stein, M. B. (2007). Psychometric analysis and refinement of the Connor-Davidson resilience scale (CD-RISC): Validation of a 10-item measure of resilience. Journal of Traumatic Stress, 20(6), 1019-1028. https://doi.org/10.1002/jts.20271

Castro, J., Pipa, J., Renner, C., O`Donnell, M. \& Cefai, C. (2018).

Enhancing Teacher Resilience Through Face-to-Face Training: Insights from the ENTREE Project. En Wosnitza, M., Peixoto, F., Beltman, S. \& Mansfield, C. F. (Eds.), Resilience in Education. Concepts, Contexts and Connections (pp. 255-274). Springer.

Cazalla-Luna, N., y Molero, D. (2016). Inteligencia emocional percibida, disposición al optimismo-pesimismo, satisfacción vital y personalidad 
de docentes en su formación inicial. Revista de Investigación Educativa, 34(1), 241-258. https://doi.org/10.6018/rie.34.1.220701

Cheung, F., \& Lucas, R. E. (2014). Assessing the validity of single-item life satisfaction measures: results from three large samples. Quality of Life Research, 23(10), 2809-2818. https://doi.org/10.1007/s11136-014$0726-4$

Choi, S. (2018). The roles of media capabilities of smartphone-based SNS in developing social capital. Behaviour \& Information Technology, 38(6), 609-620. https://doi.org/10.1080/0144929x.2018.1546903

Cleland, C., McKimm, J., Fuller, R., Taylor, D. \& Janczukowicz, J. (2020). Adapting to the impact of COVID-19: Sharing stories, sharing practice. Medical Teacher.

https://doi.org/10.1080/0142159X.2020.1757635

Coleman, J. (1988). Social capital in the creation of human capital. American Journal of Sociology, 94, 95-120.

Connor, K. M., \& Davidson, J. R. T. (2003). Development of a new resilience scale: The Connor-Davidson Resilience Scale (CD-RISC). Depression and Anxiety, 18(2), 76-82. https://doi.org/10.1002/da.10113

De la Fuente, J. \& Lozano-Díaz, A. (2010). Assessing self-regulated learning in early childhood education: Difficulties, needs and prospects. Psicothema, 22(2), 278-283.

Denovan, A., y Macaskill, A. (2017). Stress and subjective well-being among first year UK undergraduate students. Journal of Happiness Studies, 18(2), 505-525. https://doi.org/10.1007/s10902-016-9736-y

Elliott, J. R., Haney, T. J. \& Sams-Abiodun, P. (2010) Limits to social capital: Comparing network assistance in two New Orleans neighborhoods devastated by hurricane Katrina, The Sociological Quarterly, 51(4), pp. 624-648. https://doi.org/10.1111/j.15338525.2010.01186.x

Faucher, K. X. (2018). Social capital online: Alienation and accumulation. University of Westminster Press.

Fergus, S., \& Zimmerman, M. A. (2005). Adolescent resilience: A framework for understanding healthy development in the face of risk. Annual Review of Public Health, 26, 399-419. https://doi.org/10.1146/annurev.publhealth.26.021304.144357 
Fernández-Martínez, E., Andina-Díaz, E., Fernández-Peña, R., GarcíaLópez, R., Fulgueiras-Carril, I., Liébana-Presa, C. (2017). Social Networks, Engagement and Resilience in University Students. Environmental Research and Public Health, 14.

https://doi.org/10.3390/ijerph14121488

Fernández-Prados, J. S., Cuenca-Piqueras, C., \& González-Moreno, M. J. (2018). International public opinion surveys and public policy in Southern European democracies. Journal of International and Comparative Social Policy, 35(2), 227-237. https://doi.org/10.1080/21699763.2018.1535997

Fernández-Prados, J. S. \& Lozano-Díaz, A. (2020). Estudio del impacto del confinamiento en estudiantes universitarios y su satisfacción vital, resiliencia y capital social online, Mendeley Data, V1, http://dx.doi.org/10.17632/zb62c5snkm.1

Fukuyama, F. (1995). Trust: The social virtues and the creation of prosperity. Free Press.

González-Muzzio, C. (2013). El rol del lugar y el capital social en la resiliencia comunitaria posdesastre. Aproximación mediante un estudio de caso después del terremoto del 23/F. EURES, Revista Latinoamericana de Estudios Urbanos Regionales, 39(117), 25-48. http://dx.doi.org/10.4067/S0250-71612013000200002

Grottke, M., Hacker, J. V., \& Durst, C. (2018). Which factors determine our online social capital? An analysis based on structural equation modelling. Australasian Journal of Information Systems, 22, 1-36. https://doi.org/10.3127/ajis.v22i0.1656

Gutiérrez, M. y Romero, I. (2014). Resiliencia, bienestar subjetivo y actitudes de los adolescentes hacia el consumo de drogas en Angola. Anales de Psicología, 30(2), 608 - 619.

Hazel, G. (2018). Finding a Place for Resilience in Teacher Preparation. En Wosnitza, M., Peixoto, F., Beltman, S. \& Mansfield, C. F. (Eds.), Resilience in Education. Concepts, Contexts and Connections (pp. 221-236). Springer.

Heidari, E., Salimi, G., \& Mehrvarz, M. (2020). The influence of online social networks and online social capital on constructing a new graduate students' professional identity. Interactive Learning Environments, 1-18. https://doi.org/10.1080/10494820.2020.1769682 
Lee, J. (2019). Municipal-level determinants of suicide rates in South Korea: exploring the role of social capital and local government policies. Journal of Asian Public Policy, 1-18 https://doi.org/10.1080/17516234.2019.1565336

Lin, N. (1999). Building a Network Theory of Social Capital. Connections, 22(1), 28-51.

López, S., Civís, M. \& Molina, J.L. (2018). La influencia del capital social en el desarrollo profesional de maestros noveles: Una aproximación con métodos mixtos desde el análisis de redes sociales. Profesorado. Revista de currículum y formación del profesorado, 22(2), 111-132.

Martínez-Martí, M.L., y Ruch, W. (2017). Character strengths predict resilience over and above positive affect, self-efficacy, optimism, social support, self-esteem, and life satisfaction. The Journal of Positive Psychology, 12, 110-119.

https://doi.org/10.1080/17439760.2016.1163403

McGinnis, D. (2018). Resilience, Life Events, and Well-Being During Midlife: Examining Resilience Subgroups. Journal of Adult Development, 25,198-221. https://doi.org/10.1007/s10804-018-9288$\mathrm{y}$

Membiela, M. (2016). La Teoría del Capital Social. Editorial Camiño do Faro, S. L.

Membiela, M., Sánchez, E. \& Pena, J.A. (2019). La interrelación entre el "capital humano" y el "capital social". Una aproximación al caso español. ARoEc. Atlantic Review of Economics, 2, Número Especial EDaSS VII International Conference. ARoEc:

http://www.aroec.org/ojs/index.php/ARoEc/article/view/96

Moreno, R. \& Saiz, C. (2014). Factores resilientes en los futuros maestros. International Journal of Developmental and Educational Psychology, 1(2), 475-488. http://dx.doi.org/10.17060/ijodaep.2014.n1.v3.525

Nahapiet, J., \& Ghoshal, S. (1998). Social capital, intellectual capital and the organizational advantage. Academy of Management Review, 23(2), 242-266.

Nguyen, T., Dao, B., Phung, D. Q., Venkatesh, S., \& Berk, M. (2013).

Online social capital: Mood, topical and psycholinguistic analysis. Interactional AAAI Conference on Weblogs and Social Media. Massachusets, USA. 
Noble, T. \& McGrath, H. (2018). Making It Real and Making It Last ${ }_{i}$

Sustainability of Teacher Implementations of a Whole-School Resilience Programme. En Wosnitza, M., Peixoto, F., Beltman, S. \& Mansfield, C. F. (Eds.), Resilience in Education. Concepts, Contexts and Connections (pp. 289-312). Springer.

Onrubia, J. (2016). Aprender y enseñar en entornos virtuales: actividad conjunta, ayuda pedagógica y construcción del conocimiento. Revista de Educación a Distancia (RED), 50(3).

http://dx.doi.org/10.6018/red/50/3

Portes, A. (1998). Capital social: sus orígenes y aplicaciones en la sociología moderna. Annual Reviews, 24, 1-24.

https://doi.org/10.1146/annurev.soc.24.1.1

Putnam, R. D. (1993). The prosperous community: Social capital and public life. American Prospect, 4, 35-42.

Putnam, R. D. (1995). Bowling alone: America's declining social capital. Journal of Democracy, 6(1), 65-78.

Putnam, R. D. (2000). Bowling alone. Simon \& Schuster.

Ramírez-Fernández, E., Ortega-Martínez, A. R., y Calero-García, M. J. (2018). El optimismo como mediador entre la resiliencia y estados afectivos en adultos mayores. Estudios de Psicología, 39(2), 275-285. https://doi.org/10.1080/02109395.2018.1486360

Ramón, J.; Llamas, F. \& Gutiérrez, M. (2019). Revisión bibliográfica y evolución del término resiliencia. Revista Educativa Hekademos, 26, 40-47.

Rouet, G., \& Pascariu, G. C. (2019). Resilience and the EU's Eastern Neighbourhood Countries. From Theoretical Concepts to a Normative Agenda. Springer.

Sandín-Esteban, M. P. \& Sánchez-Martí, A. (2015). Resiliencia y éxito escolar en jóvenes inmigrantes. Infancia y Aprendizaje: Journal for the Study of Education and Development, 38(1), 175-211. https://doi.org/10.1080/02103702.2015.1009232

Sandoval-Hernandez, A., \& Cortes, D. (2012). Factors and conditions that promote academic resilience: A cross-country perspective. Conferencia presentada en la Conferencia Anual de la Sociedad Internacional de Educación Comparada. Puerto Rico.

Secretaría General de Universidades (2019). Datos y Cifras del Sistema 
Universitario Español. Publicación 2018-2019. Secretaría General Técnica del Ministerio de Ciencia, Innovación y Universidad. http://www.educacionyfp.gob.es/dam/jcr:2af709c9-9532-414e-9badc390d32998d4/datos-y-cifras-sue-2018-19.pdf

Selby, D., \& Kagawa, F. (2014). Towards A Learning Culture of Safety and Resilience. Technical Guidance for Integrating Disaster Risk Reduction in the School Curriculum. UNESCO and UNICEF.

Silió, E. (23 de abril de 2020). Los rectores estiman que 36.000 universitarios tienen trabas técnicas para seguir las clases o examinarse. El País: https://elpais.com/sociedad/2020-04-23/losrectores-estiman-que-36000-universitarios-tienen-trabas-tecnicaspara-ver-las-clases-o-examinarse.html

Stokes, J., \& Price, B. (2017). Social media, visual culture and contemporary identity. In 11th International Multi-conference on society, cybernetics and informatics. IMSCI:

https://www.iiis.org/CDs2017/CD2017Summer/papers/EA876TF.pdf

Tomás-Miquel, J.V.; Nicolau-Juliá, D. \& Expósito-Langa, M. (2016). The social relations of university students: intensity, interaction and association with academic performance. Cultura y Educación, 28(4), 667-701. https://doi.org/10.1080/11356405.2016.1237340

Tomyn, A., y Weinberg, M. (2018). Resilience and subjective wellbeing: A psychometric evaluation in young Australian adults. Australian Psychologist, 53(1), 68-76. https://doi.org/10.1111/ap.12251

UNICEF (2019). UNICEF Education Section (2019, March). Risk-informed education programming for resilience guidance note. UNICEF.

Vizoso, C. (2019). Resiliencia, optimismo y estrategias de afrontamiento en estudiantes de Ciencias de la Educación. Psychology, Society \& Education, 11(3), 367-377. https://doi.org/10.25115/psye.v10i1.2280

Williams, D. (2006). On and Off the 'Net: Scales for Social Capital in an Online Era. Journal of Computer-Mediated Communications, 11, 593628. https://doi:10.1111/j.1083-6101.2006.00029.x

Wood, D. F. (2016). Mens sana in corpore sano: Student well-being and the development of resilience. Medical Education, 50(1), 20-23. https://doi.org/10.1111/medu.12934

Wosnitza, M., Delzepich, R., Schwarze, J., O'Donnell, M., Faust, V. \& Camilleri, V. (2018). Enhancing Teacher Resilience: From Self- 
Reflection to Professional Development. En Wosnitza, M., Peixoto, F., Beltman, S. \& Mansfield, C. F. (Eds.), Resilience in Education. Concepts, Contexts and Connections (pp. 275-288). Springer

Antonia Lozano-Díaz is a Professor at the Department of Education, Universidad de Almería, Spain

Juan Sebastián Fernández-Prados is a Professor at the Department of Geography, History and Humanities, Universidad de Almería, Spain

Victoria Figueredo Canosa is a Professor at the Department of Education, Universidad de Almería, Spain

Ana María Martínez Martínez is a Professor at the Department of Education, Universidad de Almería, Spain

Contact Address: jsprados@ual.es 